\title{
Closing in on C. difficile infection
}

ce

both new

studies yield

insights into

CDI that could

be harnessed

in the clinic

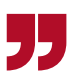

Clostridiodes difficile infection

(CDI; formerly known as Clostridium

difficile infection) poses a substantial

health-care burden, often resulting

in recurrent and difficult-to-treat

infections. Findings from two new

papers have the potential to push

forward diagnosis and treatment

of CDI, providing insights into

metabolomic networks during

human infection and new approaches

to treatment using antisporulation agents, respectively.

In the first paper, published in Journal of Clinical Investigation, Jeffrey Henderson and colleagues examined faecal metabolomes of a cohort of 186 hospitalized patients with CDI. "We had the opportunity to bring together a remarkable interdisciplinary team to see if metabolomic profiling of patients might yield useful insights into CDI," explains Henderson.

The researchers found that shifts in faecal metabolomes could distinguish patients with CDI from those with non-C. difficile diarrhoea or those only colonized by $C$. difficile. Numerous CDI-associated metabolites were identified, and of 2,463 features detected in the cohort, 43 had some ability to resolve CDI from uncolonized controls. Patients

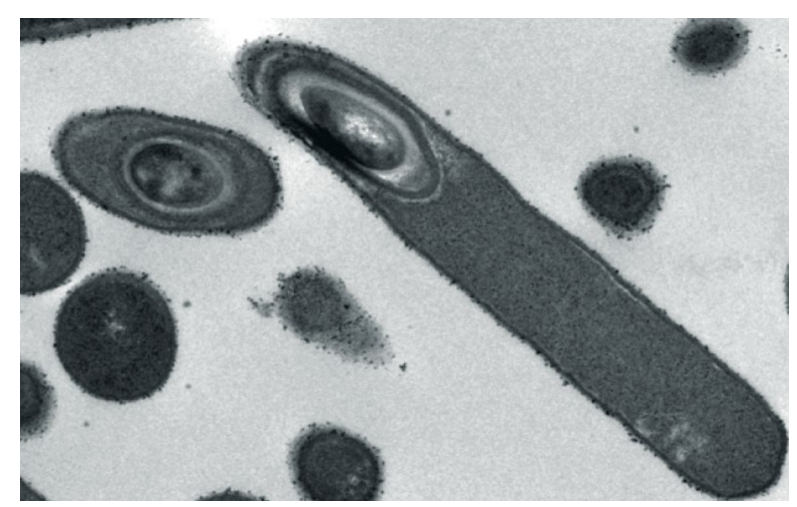

Transmission electron microscopy image of $C$. difficile spore. Image courtesy of Dena Lyras, Monash University, Australia. with CDI had a distinct chemical signature of Stickland amino acid fermentation, which was suggestive of $C$. difficile preferentially catabolizing branched-chain amino acids during CDI. Noncanonical, unsaturated bile acids (such as cholenoic acid) were depleted in patients with CDI and were more abundant in the non-CDI group.

"Comparative metabolomic profiling resolved a diverse group of metabolites associated with CDI," says Henderson. "Metabolite indices derived from these results performed well as a prototype diagnostic and may provide clinically useful information where existing diagnostics based on organism detection or faecal toxin disagree."

In the second paper, published in Nature Microbiology, the focus was on C. difficile sporulation after an unexpected observation in the lab led to the beginning of a new project. "The cephamycin cefoxitin is routinely used in selective diagnostic media for $C$. difficile, because the vegetative cell form is resistant to this antibiotic. We tried growing C. difficile on this medium to produce spores and unexpectedly we could not produce them," remarks author Dena Lyras. Realising that cefoxitin was blocking sporulation, Lyras and colleagues sought to understand the mechanism underlying this phenotype.

The investigators found that cephamycins were reducing the number of spores being produced (by 100-fold to 10,000-fold), and specifically targeted spore-specific penicillin-binding proteins (CdSpoVD, CdPGT and CdDacF). Furthermore, cephamycins were shown to have broad anti-sporulation effects, as in vitro experiments confirmed that cephamycins inhibited sporulation in other spore-forming pathogens, including Bacillus cereus.

Crucially, in a mouse model of CDI disease relapse, combined treatment with vancomycin (current standard-of-care antibiotic) and a cephamycin (cefotetan) prevented disease recurrence. In this model, mice receiving only vancomycin succumbed to recurrent disease 4-5 days after vancomycin was stopped, whereas those co-treated with vancomycin plus cefotetan had either delayed relapse after treatment was stopped (at 7.5-8.5 days with $30 \mu \mathrm{g} / \mathrm{ml}$ cefotetan) or survived to the end of the experiment (21 days with $50 \mu \mathrm{g} / \mathrm{ml}$ cefotetan).

"We identified the targets of the cephamycins and showed that these targets are common between spore-forming bacteria," notes Lyras. "This approach could work for other pathogenic microbes such as $B$. cereus (a food poisoning pathogen) and Bacillus anthracis (a bioterrorism agent)," she adds.

Although more research is necessary, both new studies yield insights into CDI that could be harnessed in the clinic. "An immediate application of the work is to assess its value in improving C. difficile diagnostics, particularly in discerning when patients benefit from C. difficile-directed antimicrobial therapy," notes Henderson. Lyras is also hopeful that their work could have clinical impact.

"The availability of a treatment to reduce spore shedding and environmental contamination, and therefore patient transmission, would be welcome by infectious disease physicians and hospitals," she concludes.

Katrina Ray

ORIGINAL ARTICLES Robinson, J. I. et al. Metabolomic networks connect host-microbiome processes to human Clostridiodes difficile infections. J.Clin. Invest. https://doi.org/10.1172/JCl126905 (2019) | Srikhanta, Y. N. et al. Cephamycins inhibit pathogen sporulation and effectively treat recurrent Clostridiodes difficile infection. Nat. Microbiol. https://doi.org/10.1038/s41564019-0519-1 (2019) 